\title{
EFEITO DA UMIDADE E DA TEMPERATURA NO PROCESSAMENTO DE FARINHA DE BANANA VERDE (Musa acuminata, GRUPO AAA) POR EXTRUSÃO TERMOPLÁSTICA
}

\author{
LAURA L.G. TORRES* \\ AHMED A. EL-DASH ${ }^{* *}$ \\ CARLOS W.P. CARVALHO*** \\ JOSÉ L.R. ASCHERI $\left.\right|^{* * * *}$ \\ ROGÉRIO GERMANI I**** \\ MARIANA MIGUEZ ${ }^{* \star * * *}$
}

\begin{abstract}
Testou-se a utilização de farinha de banana da variedade Nanicão no processo de extrusão termoplástico, visando analisar as características dos produtos obtidos em função das variáveis umidade e temperatura de extrusão. Os experimentos variaram em torno dos pontos centrais de umidade e temperatura de $20 \%$ e $150^{\circ} \mathrm{C}$, respectivamente, observando-se o efeito conjugado dessas variáveis sobre as características físicas e físico-químicas dos produtos extrusados. O processo de extrusão propiciou grande degradação do amido de banana e as amostras apresentaram elevado grau de gelatinização em todas as condições estudadas. O índice de solubilidade aumentou com a elevação da temperatura e diminuição da umidade. Verificou-se maior viscosidade de pasta a frio e a quente com aumento da umidade da matéria-prima. As maiores taxas de expansão foram obtidas com temperaturas mais elevadas e umidades mais baixas. A energia mecânica específica foi reduzida conforme o aumento da umidade e da temperatura de extrusão. Confirmou-se a quebra da molécula de amido sob as condições de extrusão pela redução do conteúdo de amido das amostras.
\end{abstract}

PALAVRAS-CHAVE: FARINHA DE BANANA VERDE; PRODUTOS EXTRUSADOS.

* $\quad$ Mestre em Ciência e Tecnologia de Alimentos, Departamento de Ciência e Tecnologia de Alimentos (DTA), Universidade Federal Rural do Rio de Janeiro (UFRRJ), Seropédica, RJ.

** Professor Titular, DTA, Faculdade de Engenharia de Alimentos (FEA), Universidade Federal de Campinas (UNICAMP), Campinas, SP (e-mail: ahmed@fea.unicamp.br).

*** Doutor em Ciência e Tecnologia de Alimentos, pesquisador da Embrapa Agroindústria de Alimentos, Rio de Janeiro, RJ (e-mail: cwpiler@ctaa.embrapa.br).

**** Doutor em Tecnologia de Cereais, pesquisador da Embrapa Agroindústria de Alimentos, Rio de Janeiro, RJ (e-mail: ascheri@ctaa.embrapa.br).

***** Doutor em Ciência e Tecnologia de Cereais, pesquisador da Embrapa Agroindústria de Alimentos, Rio de Janeiro, RJ (e-mail: germani@ctaa.embrapa.br).

***** Bolsista do CNPq, estudante de engenharia química da Universidade Federal do Rio de Janeiro (UFRJ), Rio de Janeiro, RJ (e-mail: nanamiguez@ig.com.br). 


\section{INTRODUÇÃO}

O Brasil, um dos maiores produtores mundiais de banana, exporta apenas cerca de $1 \%$ do total cultivado. Isso se deve à baixa de qualidade do produto, à obtenção de melhor qualidade por outras regiões produtoras do mundo, bem como pelo alto índice de perdas na produção (BOTREL, FREITAS-SILVA e BITTENCOURT, 2001). A industrialização da banana apresenta-se como forma de minimizar as perdas da fruta "in natura", de estimular o seu cultivo e de incentivar as exportações da fruta industrializada.

A banana é ofertada no mercado interno em quantidade e qualidade de janeiro a dezembro. Pode ser consumida em até 25 dias após sua colheita, dependendo das condições de transporte, armazenamento e comercialização. A banana, produto relativamente barato, é em geral consumida longe dos locais de produção. Tal fato onera seu preço pelos custos de embalagem, conservação e transporte (CAMPOS, VALENTE e PEREIRA, 2003).

A extrusão termoplástica representa uma das inovações fundamentais em tecnologia de alimentos. Esse processo permite a cocção de farinhas de forma contínua (milho, mandioca, arroz, soja e outras) em tempo relativamente curto (alguns segundos), modificando sua textura e formato. O processo, que combina atrito mecânico e aquecimento, permite a gelatinização do amido e a desnaturação da proteína, formando massa plástica que pode ser moldada nas mais diferentes formas (HARPER, 1992).

No âmbito dos produtos industrializados de banana detectou-se interesse pela fabricação de farinha, especialmente, de bananas verdes. Tais farinhas com elevados teores de amido têm no extrusor, equipamento versátil e acessível, uma opção para seu processamento. Por outro lado, não se encontra na literatura consultada trabalhos científicos que tratem especificamente do processamento de farinha de banana por extrusão termoplástica.

Obteve-se farinha de banana verde para testar a viabilidade da utilização dessa matéria-prima no processo de extrusão termoplástica, analisandose o efeito da umidade de processamento e da temperatura do extrusor sobre as características dos produtos extrusados. 


\section{MATERIAL E MÉTODOS}

\subsection{MATERIAL}

Foram utilizadas bananas do cultivar Nanicão, triplóide de Musa acuminata (AAA), do subgrupo Cavendish, procedentes da região de Paracambi, estado do Rio de Janeiro. O processamento das bananas ocorreu no prazo máximo de 24 horas após a colheita.

Nas determinações químicas foram utilizados reagentes com grau de pureza analítica. Para a determinação do grau de gelatinização empregouse a enzima glucoamilase com $44 \mathrm{U}$, fornecida pelo laboratório Okochi Ltda (São Paulo).

\subsection{MÉTODO DE OBTENÇÃO DA FARINHA DE BANANA}

Descascou-se a banana de forma manual e cortou-se a polpa em rodelas de $3 \mathrm{~mm}$ de espessura, mediante cortador doméstico de frios. As rodelas foram imersas em solução de 1000 ppm de metabissulfito de sódio, acidificada para pH 3,3 com ácido cítrico, segundo a metodologia descrita por RODRIGUEZ-SOSA et al. (1977).

A secagem ocorreu em estufa com circulação de ar marca FABBE, modelo 170, sendo as rodelas de banana dispostas em bandejas perfuradas e formando camadas de $12 \mathrm{~mm}$ de espessura. Manteve-se a temperatura de secagem a $66^{\circ} \mathrm{C}$, durante cerca de dez horas até que fosse atingida umidade final próxima de $8 \%$.

As rodelas de banana seca foram desintegradas em moinho de facas Wiley sem peneiras. Passou-se o produto da moagem em jogo vibratório de peneiras com peneira única e malha de 150 micrômetros de abertura. Separou-se a fração que passou pela peneira para ser utilizada em ensaios de extrusão por apresentar maior rendimento em sua preparação e coloração mais clara.

\subsection{EXTRUSÃO DA FARINHA DE BANANA}

As misturas de farinha de banana com vários teores de umidade foram extrusadas em extrusor de rosca simples de laboratório GNF 114/2 Brabender, com três zonas distintas aquecidas por sistema de indução elétrica. Mantiveram-se constantes a temperatura da primeira zona a $80^{\circ} \mathrm{C}$ e a taxa de alimentação a 3,6 kg.h-1 . Utilizou-se parafuso de taxa 
de compressão de 3:1, na velocidade de 100 rpm e matriz circular com $4 \mathrm{~mm}$ de diâmetro.

Os cálculos da produção foram efetuados com cinco amostras para cada tratamento e tempos de processamentos de 1 minuto. Separou-se uma parcela de cada amostra extrusada, sendo o restante seco em estufa com circulação de ar a 55ㄷ por 8 horas e depois moído em moinho de martelo.

As parcelas separadas foram empregadas na análise da taxa de expansão e os produtos moídos acondicionados em sacos plásticos para serem utilizados nas demais análises.

\subsection{DELINEAMENTO EXPERIMENTAL}

O delineamento central composto rotacional de $2^{\mathrm{a}}$ ordem e o modelo matemático superfície de resposta (COCHRAN e COX, 1957) foram empregados para o estudo das variáveis independentes, umidade da matéria-prima e temperatura, e da interação entre elas na extrusão de farinha de banana verde em 13 tratamentos. Desses, quatro resultaram da combinação dos níveis dos pontos 1 e -1 ; quatro para os níveis $\alpha$ e $-\alpha$ e cinco pontos centrais (Tabela 1).

Para a análise dos dados experimentais desenvolveu-se modelo matemático, baseado na equação de expansão de Taylor, cuja expressão para duas variáveis independentes foi:

$$
\mathrm{Y}_{\mathrm{i}}=B_{0}+B_{1} x_{1}+B_{2} x_{2}+B_{11} x_{1}^{2}+B_{22} x_{2}^{2}+B_{12} x_{1} x_{2}+\mathrm{E}
$$

\section{TABELA 1 - DELINEAMENTO EXPERIMENTAL COMPOSTO PARA DUAS VARIÁVEIS INDEPENDENTES E CINCO NÍVEIS DE VARIAÇÃO}

\begin{tabular}{lccccc}
\cline { 2 - 5 } Variável & \multicolumn{5}{c}{ Nível } \\
& $-\alpha^{\mathrm{a}}$ & -1 & 0 & +1 & $\alpha$ \\
\hline Temperatura $\left({ }^{\circ} \mathrm{C}\right), \mathrm{x}_{1}$ & 108 & 120 & 150 & 180 & 192 \\
Umidade $(\%), \mathrm{x}_{2}$ & 14,3 & 16 & 20 & 24 & 25,7 \\
\hline
\end{tabular}

${ }^{\mathrm{a}} \alpha=1,414$. 


\subsection{DETERMINAÇÃO DA COMPOSIÇÃO CENTESIMAL DA FARINHA DE BANANA}

Determinou-se a composição centesimal da farinha de banana verde e das farinhas extrusadas, medindo-se os teores de umidade pelo método 925.09 da AOAC (2000), proteína conforme método 990-03 da AOCC (2000), extrato etéreo pelo método 945-38 da AOAC (2000), cinzas de acordo com o método 923-03 da AOAC (2000), fibra bruta pelo método 978.10 da AOAC (2000) e carboidratos totais por diferença dos demais elementos da composição.

Na determinação do teor de amido efetuou-se a lavagem e filtração das amostras para separação dos compostos solúveis. Hidrolizou-se o amido, presente no resíduo insolúvel com $\mathrm{HCl}$, sendo os açúcares dosados pelo método de Muson e Walter (n. 31.038) da AOAC (1984).

A análise do dióxido de enxofre baseou-se na destilação do dióxiodo de enxofre liberado em meio ácido e atmosfera inerte com posterior oxidação a íon sulfúrico, determinado por titulação com hidróxido de sódio (IAL, 1976).

\subsection{DETERMINAÇÃO DA ENERGIA MECÂNICA ESPECÍFICA}

Calculou-se a energia mecânica específica (EME) pela seguinte expressão de acordo com SINGH e SMITH (1997):

$$
\operatorname{EME}\left(\text { W.h Kg }{ }^{-1}\right)=\frac{\operatorname{Torque}(\mathrm{Nm}) \times \operatorname{Rotação~}\left(\mathrm{s}^{-1}\right)}{\operatorname{Vazão~mássica~}\left(\mathrm{kg} \mathrm{h}^{-1}\right)}
$$

2.7 DETERMINAÇÃO QUÍMICA E FÍSICA DOS EXTRUSADOS DE BANANA

\subsubsection{Determinação da composição centesimal}

Para a determinação da composição centesimal foram escolhidas aleatoriamente três amostras com diferentes condições de extrusão, sendo empregados os métodos citados no item 2.5 para a farinha de banana. A análise de amido dos extrusados de banana também ocorreu de acordo com o método indicado no item 2.5. 


\subsubsection{Determinação da taxa de expansão}

Determinou-se a taxa de expansão do produto logo após a extrusão e antes da secagem, segundo metodologia descrita por ASCHERI e CARVALHO (1996). A expansão foi expressa como a relação entre o diâmetro do produto e o diâmetro da matriz. As medidas foram realizadas com paquímetro em 15 diferentes seções de cada amostra, sendo considerada a média aritmética dessas medidas.

\subsubsection{Determinação do grau de gelatinização}

Determinou-se o grau de gelatinização de todas as amostras pelo método de CHIANG e JOHNSON (1977). Tratou-se uma amostra (A) com $\mathrm{NaOH}$ para total gelatinização, sendo a outra amostra (B) apenas dispersa em água. Ambas foram digeridas com glucoamilase a $40^{\circ} \mathrm{C}$, durante 40 minutos, inativando-se a enzima logo a seguir com TCA a $25 \%$. Determinou-se o grau de hidrólise, medindo os açúcares redutores (expressos como glicose) por reação com ortotoluidina. As leituras de absorbância ocorreram a 630 nm e a porcentagem de gelatinização foi calculada pela seguinte fórmula:

$\%$ de gelatinização $=\frac{(B-K)}{(A-K)} \times 100$

Na qual:

A = absorbância de uma amostra de farinha com amido totalmente gelatinizado.

$\mathrm{B}$ = absorbância do amido pré-gelatinizado (amostras de farinha prégelatinizadas por extrusão).

$\mathrm{K}=$ absorbância de uma amostra de farinha intacta de banana.

\subsubsection{Determinação da viscosidade de pasta}

Determinou-se a viscosidade da farinha de banana crua e das amostras processadas utilizando viscoamilógrafo Brabender (ASCHERI e CARVALHO, 1997). As amostras foram submetidas ao aquecimento partindo-se da temperatura de $25^{\circ} \mathrm{C}$ para $95^{\circ} \mathrm{C}$ a taxa constante de elevação de $1,5^{\circ} \mathrm{C}$ por minuto. Manteve-se a temperatura de $95^{\circ} \mathrm{C}$ constante por 20 minutos e procedeu-se o resfriamento até $50^{\circ} \mathrm{C}$, com diminuição de temperatura de $1,5^{\circ} \mathrm{C}$ por minuto. 
Os amilogramas foram obtidos a partir de suspensões preparadas com $450 \mathrm{~mL}$ de água destilada e $4 \mathrm{~g}$ (b.s.) de farinha de banana. Essa proporção também foi empregada para os extrusados moídos, usando-se nesse caso agitador mecânico para facilitar a dissolução e homogeneização prévia das amostras. Somente as seguintes análises dos amilogramas foram consideradas:

- viscosidade da pasta a frio $\left(25^{\circ} \mathrm{C}\right)=$ valor da viscosidade da suspensão em unidades amilográficas (UA) no início do ciclo de aquecimento;

- viscosidade a quente = corresponde ao valor da viscosidade (em UA) quando a suspensão chega a $95^{\circ} \mathrm{C}$ no ciclo de aquecimento.

\subsection{5 Índices de absorção (IAA) e de solubilidade (ISA) em água}

A determinação dos índices de absorção e solubilidade em água seguiu a metodologia descrita por FERNANDES et al. (2002) com modificações. Em tubos de centrífuga com tampa foram colocados $1,0 \mathrm{~g}$ (b.s) de amostra e $30 \mathrm{~mL}$ de água. Os tubos foram agitados durante 30 minutos em agitador mecânico a $30^{\circ} \mathrm{C}$ e depois centrifugados a $3500 \mathrm{rpm}$ por 20 minutos. Pesou-se o gel remanescente, após retirada do sobrenadante, sendo o índice de absorção de água (IAA) calculado conforme a seguinte relação:

$$
\text { IAA }=\frac{\text { peso do gel }(\mathrm{g})}{\text { peso seco da amostra }(\mathrm{g})-\text { peso do resíduo de evaporação }(\mathrm{g})}
$$

Verteu-se o líquido sobrenadante em placa de Petri, previamente tarada, colocando-a em estufa a $105^{\circ} \mathrm{C}$ até atingir peso constante. O índice de solubilidade em água (ISA) foi determinado pela seguinte fórmula:

$$
\mathrm{ISA}=\frac{\text { peso do resíduo de evaporação }(\mathrm{g}) * 100}{\text { peso seco da } \operatorname{amostra}(\mathrm{g})}
$$

\section{RESULTADOS E DISCUSSÃO}

\subsection{COMPOSIÇÃO QUÍMICA DA FARINHA CRUA E EXTRUSADA}

Na Tabela 2 observa-se a composição química de três amostras de farinhas de banana extrusadas, escolhidas ao acaso, e a comparação desses valores com os da farinha de banana crua (antes da extrusão). A extrusão não provocou grande variação na composição das farinhas, a não ser pela substancial redução no teor de amido. Tal fato pode ser 
explicado pela possível degradação molecular do amido em compostos de menor peso molecular não-detectáveis.

\section{TABELA 2 - VALORES MÉDIOS DA COMPOSIÇÃO QUÍMICA DE AMOSTRAS DE FARINHA DE BANANA EXTRUSADA E DE FARINHA DE BANANA CRUA}

\begin{tabular}{|c|c|c|c|c|}
\hline \multirow{3}{*}{$\begin{array}{l}\text { Composição } \\
\text { (matéria seca) }\end{array}$} & \multirow{3}{*}{$\begin{array}{c}\text { Farinha de } \\
\text { banana verde } \\
\text { crua }\end{array}$} & \multicolumn{3}{|c|}{ Amostras extrusadas } \\
\hline & & \multicolumn{3}{|c|}{ Tratamentos } \\
\hline & & 7 & 12 & 13 \\
\hline Proteína (g/100 g) & 3,72 & 3,63 & 3,71 & 3,64 \\
\hline Extrato Etéreo $(\mathrm{g} / 100 \mathrm{~g})$ & 0,53 & 0,25 & 0,45 & 0,41 \\
\hline Carboidratos totais $(\mathrm{g} / 100 \mathrm{~g})$ & 91,70 & 91,80 & 90,70 & 91,30 \\
\hline Fibra bruta $(\mathrm{g} / 100 \mathrm{~g})$ & 2,01 & 1,94 & 2,74 & 2,34 \\
\hline Cinza $(g / 100 \mathrm{~g})$ & 2,00 & 2,36 & 2,33 & 2,34 \\
\hline Amido $(\mathrm{g} / 100 \mathrm{~g})$ & 87,80 & 75,60 & 75,50 & 76,20 \\
\hline
\end{tabular}

\subsection{ANÁLISE ESTATÍSTICA DOS RESULTADOS}

Os coeficientes da equação 1 (Tabela 3) foram obtidos pelo ajuste dos dados experimentais, não sendo representadas graficamente em superfície de respostas (Figuras 1 a 6) as variáveis com coeficientes de regressão inferiores a 0,75.

\section{TABELA 3 - COEFICIENTES SIGNIFICATIVOS DA EQUAÇÃO DE REGRESSÃO (eqn 1) ${ }^{1}$ PARA AS VARIÁVEIS DEPENDENTES}

\begin{tabular}{lccccccc}
\hline Coeficientes & $\begin{array}{c}\text { Viscosidade } \\
\text { a Frio }(\mathbf{c P})\end{array}$ & $\begin{array}{c}\text { Viscosidade } \\
\text { a Quente } \\
\text { (cP) }\end{array}$ & IAA & ISA & $\begin{array}{c}\text { Grau de } \\
\text { gelatinização }\end{array}$ & Expansão & EME $\left(\mathrm{Wh} \mathrm{Kg}^{-1}\right)$ \\
\hline $\mathrm{B}$ & $278.83^{\star \star \star}$ & $21.83^{\star \star \star}$ & $12.81^{\star \star \star}$ & $33.49^{\star \star \star}$ & $122.98^{\star \star \star}$ & $1.64^{\star \star \star}$ & $11.23^{\star \star \star}$ \\
$\mathrm{B}$ & $92.82^{\star \star \star}$ & $15.82^{\star \star \star}$ & N.S. & $-9.89^{\star \star}$ & $-9.40^{\star \star \star}$ & $-0.37^{\star \star \star}$ & $-7.19^{\star \star \star}$ \\
$\mathrm{B}_{2}^{1}$ & N.S. & $-12.28^{\star \star}$ & $1.14^{\star \star}$ & $2.02^{\star}$ & $8.31^{\star \star \star}$ & $-0.36^{\star \star \star}$ & $-5.62^{\star \star}$ \\
$\mathrm{B}_{11}$ & N.S. & N.S. & $1.06^{\star}$ & N.S. & N.S. & N.S. & $4.19^{\star}$ \\
$\mathrm{B}_{22}$ & $-103.87^{\star \star \star}$ & N.S. & N.S. & N.S & $-7.64^{\star \star}$ & $0.18^{\star \star \star}$ & N.S. \\
$B_{12}$ & N.S. & $-12.50^{\star}$ & N.S. & N.S. & $-9.05^{\star \star}$ & $0.18^{\star \star}$ & $7.63^{\star \star}$ \\
$\mathrm{R}^{2}$ & 0.91 & 0.81 & 0.61 & 0.95 & 0.88 & 0.96 & 0.83 \\
\hline
\end{tabular}

****** significativos a $p \leq 0,1, p \leq 0,05$ e $p \leq 0,01$, respectivamente.

N.S.= Não-significativo.

${ }^{1} \mathrm{x} 1=$ temperatura $\left({ }^{\circ} \mathrm{C}\right)$ e $\mathrm{x} 2=$ umidade (\% b.u. $)$.

IAA = índice de absorção de água.

ISA = índice de solubilidade em água.

$\mathrm{EME}=$ energia mecânica específica. 


\subsubsection{Viscosidade de pasta a frio}

A viscosidade da suspensão de farinha em água a $25^{\circ} \mathrm{C}$ é considerada a frio. Essa propriedade, importante na preparação de alimentos instantâneos, indica a capacidade das farinhas em absorver água a temperatura ambiente e formar pasta, gel ou líquido viscoso. Na Figura 1 pode-se verificar que a temperatura e a umidade foram altamente significativas, sendo o coeficiente de determinação $\left(R^{2}\right)$ superior a $90 \%$ da variação da resposta. Embora a temperatura afete a viscosidade a frio, a umidade exerce maior influência sobre essa variável, o que também foi constatado por WHALEN et al. (1997) em experimentos com grits de milho.

A redução rápida da viscosidade, com o aquecimento e a agitação, caracteriza o completo rompimento da estrutura dos grânulos de amido. Não houve picos na faixa de $60-70^{\circ} \mathrm{C}$ como ocorreu com a farinha crua, o que também confirma a ausência de grânulos intactos.

\section{FIGURA 1 - EFEITO DA TEMPERATURA E DA UMIDADE NA VISCOSIDADE A FRIO (25ㄷ)}

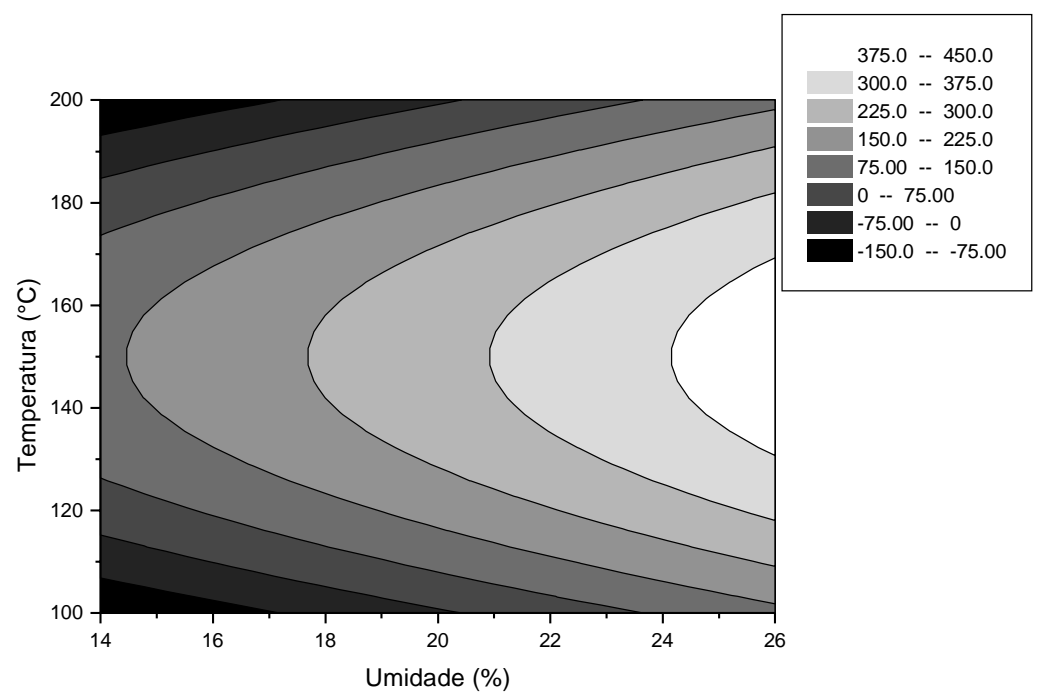




\subsubsection{Viscosidade a quente $\left(95^{\circ} \mathrm{C}\right)$}

A viscosidade a quente indica as possibilidades de utilização da farinha extrusada. Torna-se importante quando, por exemplo, a farinha for destinada ao preparo de sopas, tortas ou outros alimentos em que é necessário conservar a viscosidade em temperaturas acima da ambiente.

De acordo com HAASE, NINTUS e WEIPERT (1995), a viscosidade a quente está relacionada com o nível de degradação sofrido pelo grânulo de amido. Após destruição de sua estrutura, o grânulo perde sua capacidade de inchar quando aquecido em água e apresenta baixa viscosidade a $95^{\circ} \mathrm{C}$. Comportamento semelhante foi observado em todas as amostras extrusadas de farinha de banana verde.

A temperatura e a interação da temperatura-umidade foram significativas para os valores de viscosidade a $95^{\circ} \mathrm{C}(\mathrm{P}<0,05)$. $\mathrm{O}$ modelo adotado explicou $81,2 \%\left(R^{2}\right)$ da variação da resposta.

Altas umidades e baixas temperaturas, indicativas de tratamento térmico menos severo, forneceram as maiores viscosidades a $95^{\circ} \mathrm{C}$ (Figura 2 ).

\section{FIGURA 2 - EFEITO DA TEMPERATURA E DA UMIDADE NA VISCOSIDADE DE PASTA A $95^{\circ} \mathrm{C}$}

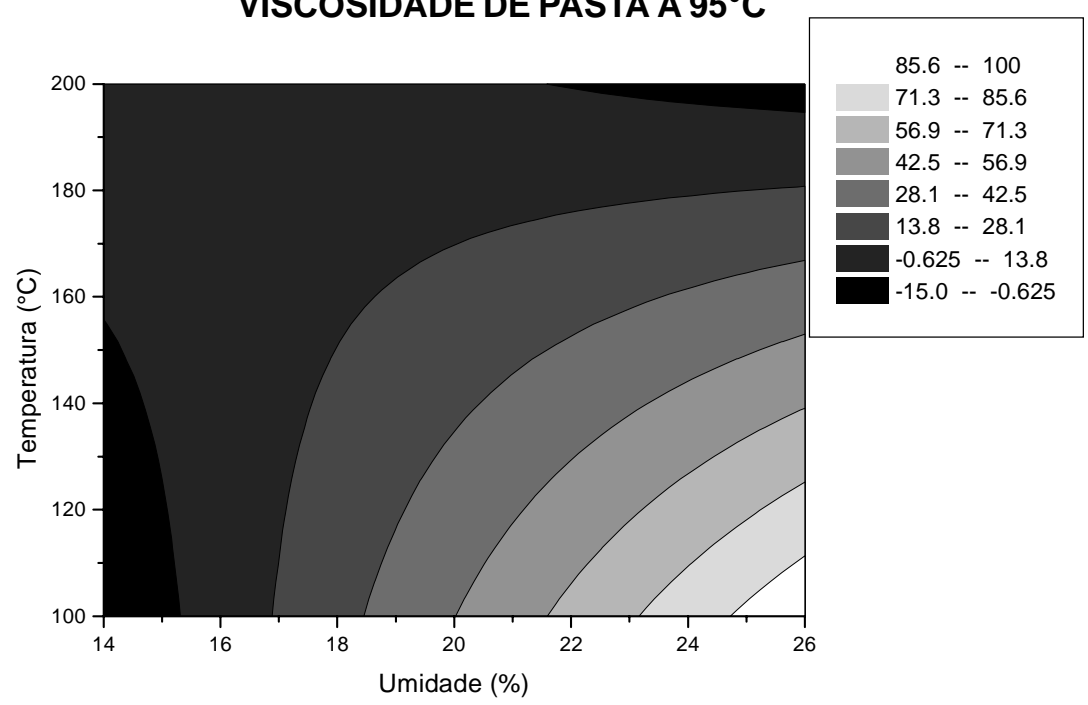


SILVA et al. (2004) observaram que a degradação dos grânulos de amido aumenta em baixa umidade, pois o atrito mecânico no parafuso é maior. Isto se reflete em menores condições de entumescimento dos grânulos, diminuindo a viscosidade. Da mesma forma, o atrito mecânico diminuiu com alta umidade havendo menor destruição dos grânulos.

\subsection{EFEITO DAS VARIÁVEIS DE TEMPERATURA E UMIDADE NO IAA E ISA}

A absorção de água, considerada como medida do grau de modificação de amido, é outra propriedade indicativa das possibilidades de utilização das farinhas extrusadas. Alta capacidade de absorção de água é desejável para o preparo de sopas, mingaus e pudins instantâneos. Somente os grânulos de amido danificados, tendo grupos hidrofílicos disponíveis, podem absorver água em temperatura ambiente. Quando, o amido é submetido à ação da energia mecânica e/ou térmica de maior grandeza, o IAA decresce indicando destruição da integridade molecular do amido (SRIBURI e HILL, 2000).

Obteve-se índice de absorção de água $(15,86)$ mais elevado para os extrusados de farinha de banana com maior teor de umidade $(25,7 \%)$ e apesar do baixo coeficiente de regressão $\left(R^{2}=0,61\right)$ não foi observada correlação direta entre a umidade e o IAA. A variação do IAA em função da umidade e da temperatura de extrusão mostrou-se significativa ao nível de $5 \%$ de probabilidade. O coeficiente de determinação na análise de regressão foi baixo, em torno de $60 \%$.

O ISA representa a conseqüência da degradação total sofrida pelo grânulo de amido, ou seja, a soma dos efeitos de gelatinização e dextrinização. De acordo com HASHIMOTO et al. (2002) e ASCHERI e CARVALHO (1996), o ISA aumenta com a severidade do tratamento recebido. É de se esperar, portanto, aumento no ISA com a solubilização e rompimento dos grânulos de amido. Pelos resultados da análise de regressão, com as variáveis significativas, constatou-se que a umidade e a temperatura afetaram essa propriedade. O modelo adotado explicou 95,3\% da variação da resposta $(\mathrm{P}<0,05)$.

O diagrama de superfície de resposta, correspondente ao modelo, indicou que a diminuição do teor de umidade e a elevação da temperatura aumenta o ISA (Figura 3). Nas condições de extrusão utilizadas a combinação de baixo teor de umidade e alta temperatura resultou em maior degradação dos grânulos de amido, contribuindo assim para o aumento do valor do ISA. 


\section{FIGURA 3 - EFEITO DA TEMPERATURA E DA UMIDADE SOBRE $O$}

ISA

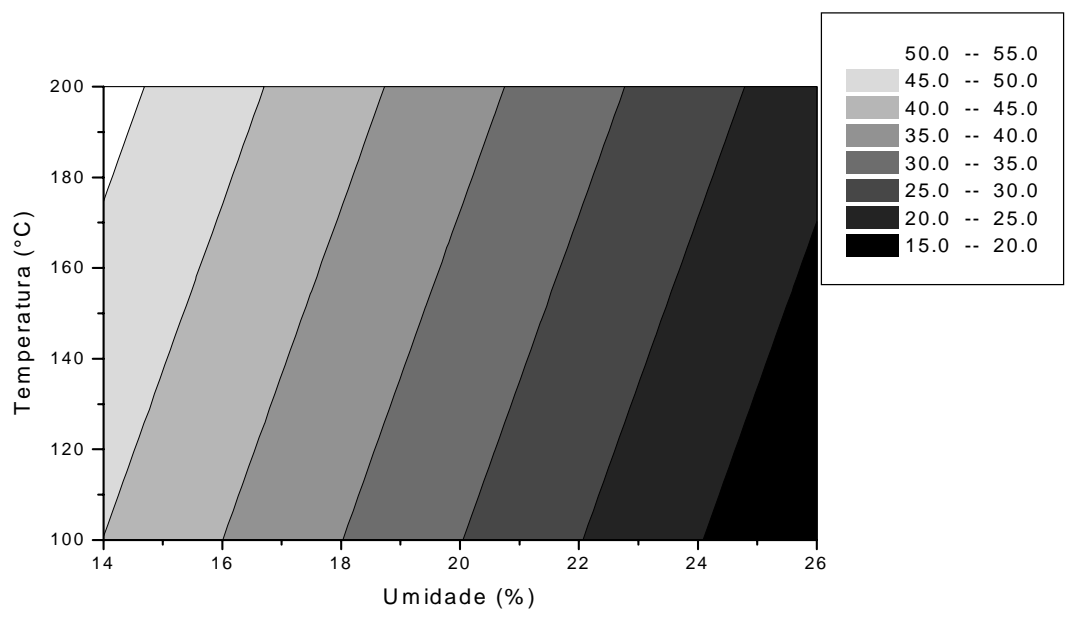

\subsection{EFEITO DO TEOR DE UMIDADE DA FARINHA E DA TEMPERATURA DE EXTRUSÃO NO GRAU DE GELATINIZAÇÃO}

Quase todos os valores obtidos para o grau de gelatinização das amostras extrusadas ultrapassaram $100 \%$ de gelatinização. Pode-se supor que a farinha de banana crua tratada com $\mathrm{NaOH}$, a fim de ser gelatinizada e usada como referencial (padrão) não foi totalmente gelatinizada. É mais provável que mesmo as condições mais brandas da extrusão tenham destruído a estrutura do amido, tornando-o bem mais susceptível ao ataque enzimático e possibilitando valores elevados de gelatinização em relação ao padrão (Figura 4).

Os maiores graus de gelatinização ocorreram com as combinações de alta temperatura e baixa umidade (Figura 4). Os resultados encontrados estão de acordo com os otidos por ZHENG e WANG (1994) para a gelatinização de amido de milho extrusado. Esses verificaram maior grau de gelatinização com valores extremos opostos para as variáveis umidade e temperatura.

Pela análise estatística verifica-se que a umidade, a temperatura e a interação entre essas variáveis afetaram significativamente a gelatinização do amido. O modelo da equação de regressão explicou $88,56 \%$ da variação da resposta. 
GRAFELMAN e MEAGHER (1995) afirmaram que a extrusão facilita a ação enzimática sobre o amido, provavelmente devido à gelatinização, à inativação de inibidores endógenos das amilases, ao rompimento da estrutura celular e/ou redução do tamanho da superfície exposta das moléculas.

\section{FIGURA 4 - EFEITO DA TEMPERATURA E DA UMIDADE SOBRE $O$ GRAU DE GELATINIZAÇÂO}

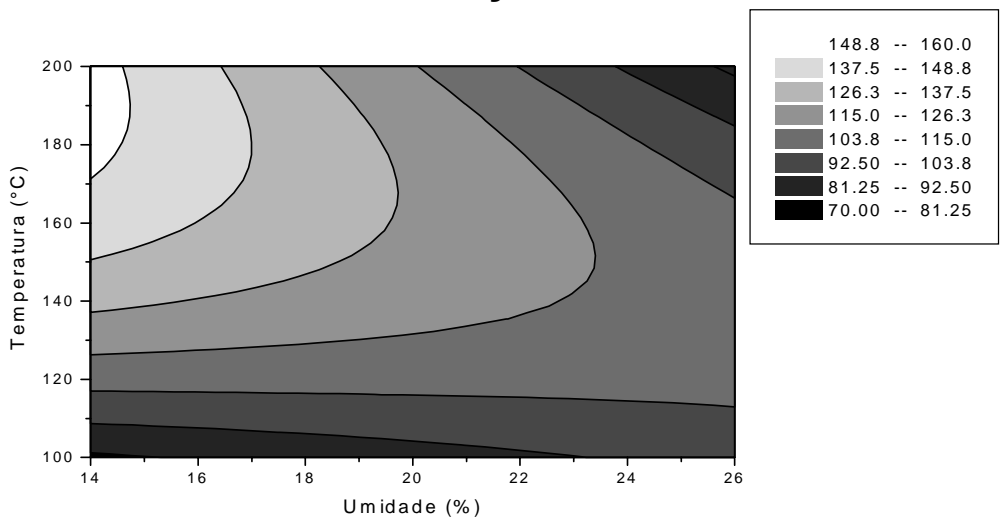

\subsection{EFEITO DO TEOR DE UMIDADE DA FARINHA E DA TEMPERATURA DE EXTRUSÃO NA TAXA DE EXPANSÃO}

A expansão é usualmente expressa pela relação entre a área da seção transversal do extrusado e a área da matriz, ou pela relação entre os diâmetros do produto extrusado e da matriz sendo essa última adotada neste trabalho.

Na Figura 5 pode-se observar a superfície de resposta correspondente à variação da expansão em função do teor de umidade da matéria-prima e da temperatura de extrusão. A umidade, a temperatura e a interação umidade-temperatura contribuíram significativamente para o modelo. $O$ coeficiente de determinação foi elevado, explicando $96,1 \%$ da variação da resposta. Obteve-se a menor taxa de expansão com a umidade mais alta combinada com temperatura elevada $\left(25,7 \%\right.$ e $\left.150^{\circ} \mathrm{C}\right)$. Tal resultado está de acordo com as variações observadas por diversos autores (LAM e FLORES, 2003; ZASYPKIN e LEE, 1998; CAI e DIOSADY, 1993). 
Baixa umidade e o elevada temperatura não aumentaram a taxa de expansão, sendo observada maior taxa de expansão em umidade e temperatura baixas $\left(16 \%\right.$ e $\left.120^{\circ} \mathrm{C}\right)$. Isto deve-se ao fato de que em temperaturas superiores a $120^{\circ} \mathrm{C}$, a estrutura do extrusado não foi capaz de suportar expansão maior.

DOGAN e KARWE (2003) constataram redução da expansão radial de extrusados de quinoa em temperaturas superiores a $150^{\circ} \mathrm{C}$. O decréscimo em altas temperaturas foi atribuído à excessiva quebra molecular do amido, resultando em enfraquecimento da matriz amilácea na retenção da evaporação da água. Tal fato também foi confirmado por GUJRAL, SINGH e SINGH (2001) que estudaram o efeito do tamanho de partícula e da umidade na extrusão de grits de milho. Nos extrusados expandidos de milho processados em temperaturas próximas a $200^{\circ} \mathrm{C}$, a evaporação ocorria com tal violência que rompia a estrutura do material reduzindo a expansão radial dos extrusados. Por outro lado, observaram que a expansão decrescia rapidamente quando o conteúdo de umidade de processamento aumentava. Baixa expansão foi verificada em altas umidades como conseqüência do colapso da estrutura, causado pela grande quantidade de vapor eliminada no momento que o extrusado deixa a matriz do extrusor (CAI e DIOSADY, 1993).

\section{FIGURA 5 - EFEITO DAS VARIÁVEIS TEMPERATURA E UMIDADE NA TAXA DE EXPANSÃO}

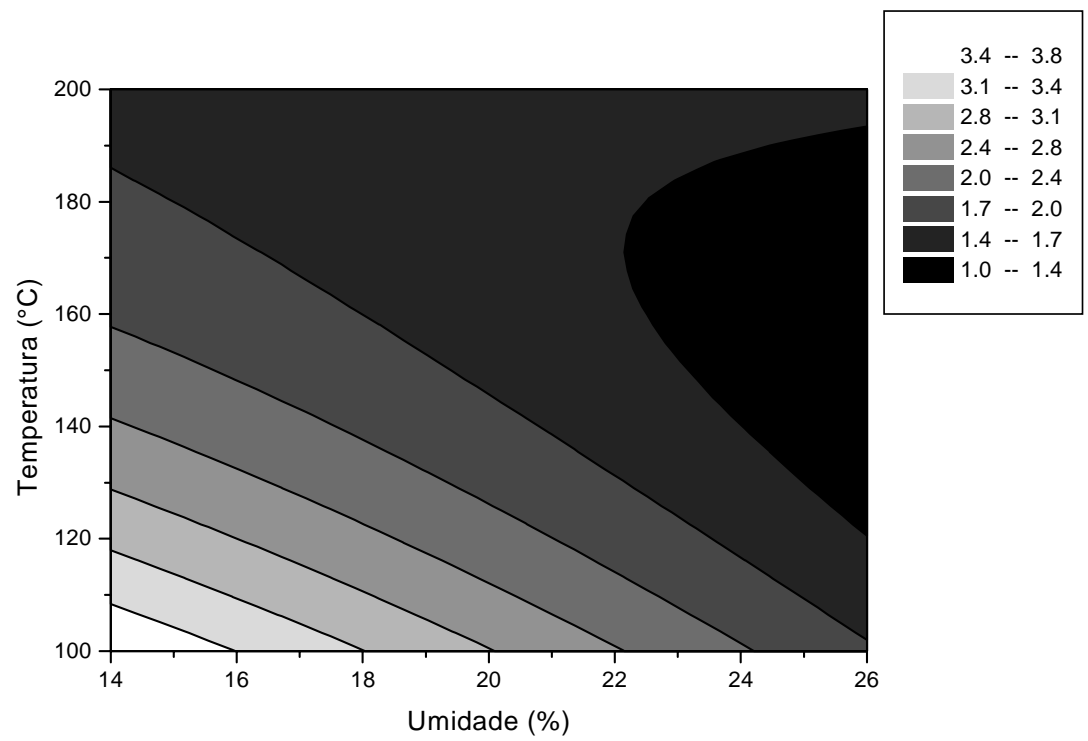


3.7 EFEITO DO TEOR DE UMIDADE DA FARINHA E DA TEMPERATURA DE EXTRUSÃO NA ENERGIA MECÂNICA ESPECÍFICA (EME)

A energia mecânica específica torna-se importante na análise dos extrusados de amido (CHANG et al., 1999) por permitir monitorar variações nos parâmetros do processo e da formulação (ONWULATA et al., 2001).

A umidade foi a variável mais significativa $(P<0,001)$, seguida pela interação temperatura e umidade $(P<0,01)$. O coeficiente de determinação indicou que $80,5 \%$ da variação da resposta foi explicada pelo modelo representado na Figura 6. O aumento da EME observado com baixa umidade explica-se pela elevação do atrito mecânico ao deslocar o material fundido dentro do extrusor até a saída da matriz. Ao aumentar a temperatura a viscosidade do material diminui e o fluxo aumenta, reduzindo a energia mecânica específica. A água exerce ação lubrificante com maior teor de umidade, o fluxo se torna mais fácil e ocasiona diminuição da EME mesmo em baixas temperaturas (WILLET, MILLARD e JASBERG, 1997).

\section{FIGURA 6- EFEITO DA UMIDADE DA MATÉRIA-PRIMA E TEMPERATURA DE EXTRUSÃO NA ENERGIA MECÂNICA ESPECÍFICA (EME)}

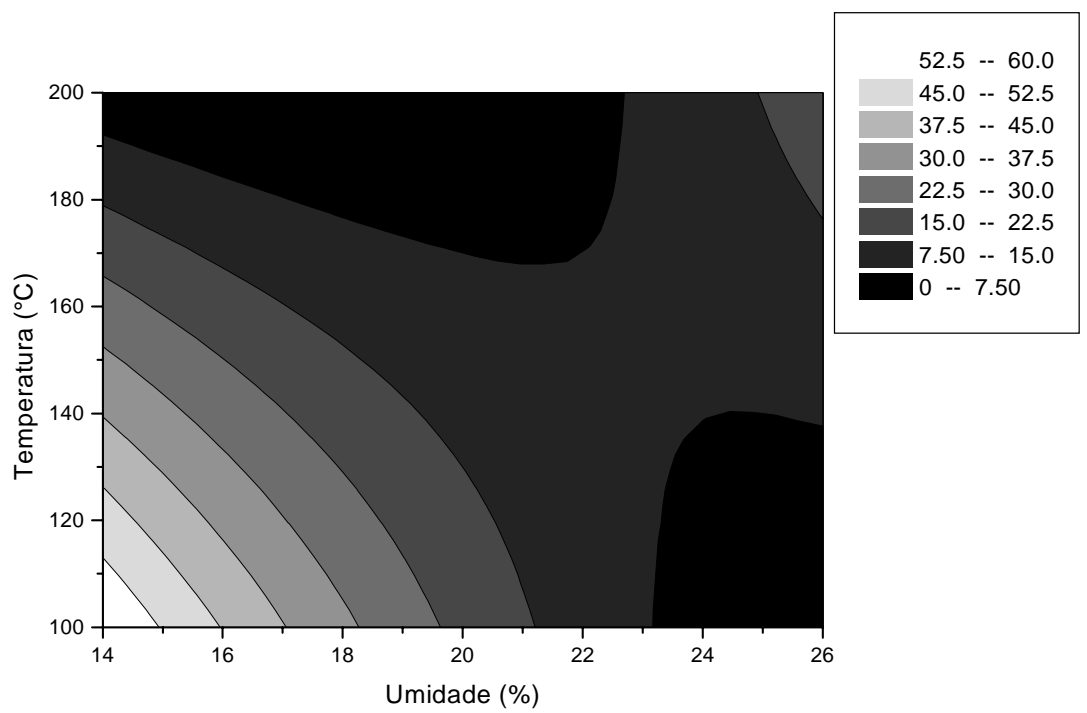




\title{
4 CONCLUSÃO
}

Todas as propriedades físicas nas condições estudadas foram influenciadas significativamente pelas variáveis umidade da matéria-prima e temperatura de extrusão.

Com o processamento da banana verde por extrusão, a susceptibilidade do amido à amiloglucosidase aumentou sensivelmente, até nas condições mais brandas de processamento aplicadas.

Os extrusados de farinha de banana apresentaram pequenas taxas de expansão após a extrusão. As maiores taxas foram obtidas com a combinação de baixas temperaturas (inferiores a $120^{\circ} \mathrm{C}$ ) e baixas umidades (até 16\%).

\begin{abstract}
MOISTURE CONTENT AND TEMPERATURE EFFECTS ON THE PROCESSING OF UNRIPE BANANA FLOUR (MUsa acuminata, AAA GROUP) BY THERMOPLASTIC EXTRUSION

The utilization of Giant-Cavendish bananas flour in the thermoplastic extrusion process was tested, aiming to analyze the characteristics of the obtained products in relation to moisture content and extrusion temperature. The experiment variables were around the central points of moisture content and temperature of respectively $20 \%$ and $150^{\circ} \mathrm{C}$, being the conjugated effect of the variables on the physical and physico-chemical characteristics of the extruded products observed by surface response diagrams. The extrusion process resulted in high degradation of the banana starch and the samples showed high degree of gelatinization in all the conditions studied. Solubility index increased with temperature rise and moisture drop. Both cold and hot-paste viscosity increased with augmented moisture content. The greatest expansion ratio was obtained with higher temperatures and lower moisture contents. Specific mechanical energy was reduced with an increase of extrusion temperature and moisture. Water absorption of the extrudate flour was significantly affected by temperature and moisture. The breakdown of the starch molecules under the processing conditions was confirmed by the reduction of starch content of the samples
\end{abstract}

KEY-WORDS: UNRIPE BANANA FLOUR; EXTRUDATE PRODUCTS.

\section{REFERÊNCIAS}

1 ASCHERI, J.L.R.; CARVALHO, C.W.P. Caracterización fisico quimica de snacks de grits de maiz producidos por extrusion termoplástica. Alimentaria, v. 34, n. 273, p. 87-91, 1996.

2 ASCHERI, J.L.R; CARVALHO, C.W.P. Efecto de los parámetros de extrusion, características de pasta y textura de pellets (snacks de tercera generación) 
producidos a partir de trigo y maiz. Alimentaria, v. 35, n. 279, p. 93-98, 1997.

3 AOAC. Association of Official Analytical Chemistry. Official Methods of Analysis of the AOAC International. 14 $4^{\text {th. }}$, Virginia, 1984.

4 AOAC. Association of Official Analytical Chemistry. Official Methods of Analysis of the AOAC International. 17 $7^{\text {th }}$, Virginia, 2000.

5 BOTREL, N.; FREITAS-SILVA, O.; BITTENCOURT, A.M. Procedimentos pós-colheita. In: MATSUURA, F. C. A. U.;FOLEGATTI, M. I. S. (Ed.). Banana: pós colheita. Brasília: Embrapa Informação Tecnológica, 2001. p. 71. (Frutas do Brasil).

6 CAI, W.; DIOSADY, L.L. Modeling of Expansion and Water solubility index of wheatstarch during extrusion-cooking. Acta Alimentaria, v. 22, n. 3, p. 181-192, 1993.

7 CAMPOS, R.P.; VALENTE, J.P.; PEREIRA, W.E. Postharvest of banana "nanicão" produced in the states of Mato Grosso and Santa Catarina, marketed in Cuiabá MT. Revista Brasileira de Fruticultura, v. 25, n. 1, p. 172-174, 2003.

8 CHANG, Y.K.; MARTINEZ-BUSTOS, F.; PARK, T.S.; KOKINI, J.L. The influence of specific mechanical energy on cornmeal viscosity measured by an on-line system during twin-screw extrusion. Brazilian Journal Of Chemical Engineering, v. 16, n. 3, p. 285-295, 1999.

9 CHIANG, B.Y.; JONHSON, J. A. Measurement of total and gelatinized starch by glucoamylase and o-toluidine reagent. Ceral Chem., St. Paul, v. 54, n. 3, p. 429435, 1977.

10 COCHRAN, W.G.; COX. G.M. Some methods for the study of response surfaces. In: EXPERIMENTAL Design. $2^{\text {nd }}$ ed. New York: Jonh Wiley \& Sons, 1957, p. 335369.

11 DOGAN, H.; KARWE, M.V. Physicochemical properties of quinoa extrudates. Food Science and Technology International, v. 9, n. 2, p. 101-114, 2003.

12 FERNANDES, M.S.; WANG, S.H.; ASCHERI, J.L.R.; OLIVEIRA, M.F.; COSTA, S.P.J. Puffed extruded corn-grits and soybean mixtures for snacks use. Pesquisa Agropecuaria Brasileira, v. 10, n. p. 1459-1501, 2002.

13 GRAFELMAN, D.D.; MEAGHER, M.M. Liquefaction of starch by a single-screw extruder and post-extrusion Static-Mixer Reactor. Journal of Food Engineering, v. 24, n. 4, p. 529-542, 1995.

14 GUJRAL, H.S.; SINGH, N.; SINGH, B. Extrusion behaviour of grits from flint and sweet corn. Food Chemistry, v. 74, n. 3, p. 303-308, 2001.

15 HAASE, N.U.; MINTUS, T.; WEIPERT, D. Viscosity measurements of potato starch paste with the Rapid- Visco-Analyzer. Starch-Starke, v. 47, n. 4, p. 123-126, 1995. 
16 HARPER, J.M. A comparative analysis of single and twin-screw extruders. In: KOKINI, J. L.; HO, C.-T.; KARWE, M. V. (Ed.). Food extrusion science and technology. New York: Marcel Dekker, 1992. p. 139-148.

17 HASHIMOTO, J.M.; NABESHIMA, E.H.; CINTRA, H.S.; DIAS, A.R.G.; BUSTOS, F.M.; CHANG, Y.K. Effect of processing conditions on some functional characteristics of extrusion-cooked cassava starch/wheat gluten blends. Journal of the Science of Food and Agriculture, v. 82, n. 8, p. 924-930, 2002.

18 IAL. Instituto Adolfo Lutz. Normas analíticas do Instituto Adolfo Lutz. São Paulo, Secretaria do Estado de Saúde, 1976. v.1, p. 74-75.

19 LAM, C.D.; FLORES, R.A. Effect of particle size and moisture content on viscosity of fish feed. Cereal Chemistry, v. 80, n. 1, p. 20-24, 2003.

20 ONWULATA, C.I.; SMITH, P.W.; KONSTANCE, R.P.; HOLSINGER, V.H. Incorporation of whey products in extruded corn, potato or rice snacks. Food Research International, v. 34, n. 8, p. 679-687, 2001.

21 RODRIGUEZ-SOSA, E.J.; GONZÁLEZ, M.L.; CALONI, I.B.; PARSI-ROS, O. The preparation of green banana flour. J. Agric. Univ. Puerto Rico, v. 61, n. 4, p. 470-478,1977.

22 SRIBURI, P.; HILL, S. E. Extrusion of cassava starch with either variations in ascorbic acid concentration or $\mathrm{pH}$. International Journal of Food Science and Technology, v. 35, n. 2, p. 141-154, 2000.

23 SILVA, M.C.; THIRÉ, R.M.S.M.; PITA, V.J.R.R.; CARVALHO, C.W.P.; ANDRADE, C.T. Processamento de amido de milho em câmara de mistura. Ciência e Tecnologia de Alimentos, v. 24, n. 2, p. 303-310, 2004.

24 SINGH, N.; SMITH, A.C. A comparison of wheat starch, whole wheat meal and oat flour in the extrusion cooking process. Journal of Food Engineering, v. 34, n. 1, p. 15-32, 1997.

25 WHALEN, P.J.; BASON, M.L.; BOOTH, R.I.; WALKER, C.E.; WILLIAMS, P.J. Measurement of extrusion effects by viscosity profile using the rapid ViscoAnalyser. Cereal Food World, v. 42, n. p. 469-475, 1997.

26 WILLETT, J.L.; MILLARD, M.M.; JASBERG, B.K. Extrusion of waxy maize starch: melt rheology and molecular weight degradation of amylopectin. Polymer, v. 38, n. 24, p. 5983-5989, 1997.

27 ZASYPKIN, D.V.; LEE, T.C. Extrusion of soybean and wheat flour as affected by moisture content. Journal of Food Science, v. 63, n. 6, p. 1058-1061, 1998.

28 ZHENG, X.; WANG, S.S. Shear induced starch conversion during extrusion. Journal of Food Science, v. 59, n. 5, p. 1137-1143, 1994. 